\title{
Zur Methodik der Psychotherapie im Kindesalter mit besonderer Berücksichtigung der „Miliensuggestions-Methode“s
}

\author{
Von \\ C. Pototzky.
}

(Aus dem Kaiserin Auguste Victoria Hause zur Bekämpfung der Säuglingssterblichkeit im Deutschen Reiche, Charlottenburg. Direkt.: Prof. Dr. Langstein.)

Wenn auch heute erst ganz allmählich die psychotherapeutischen Methoden unter das Rüstzeug des Kinderarztes aufgenommen werden und sie keineswegs bisher zum Allgemeingut des Praktikers geworden sind, so liegt dies nicht zum mindesten daran, daß in den Lehrbüchern der Kinderheilkunde und selbst in den speziellen psychotherapeutischen Arbeiten von Kinderärzten meist nur in allgemeinen Angaben auf die einzelnen Methoden eingegangen wird. Man begnügt sich in der Regel mit einer Aufzählung der Fälle, die mit Wachsuggestion, Hypnose, Persuasion, Psychoanalyse, Frankschem kathartischem Verfahren usw. geheilt worden sind. Zur Nachahmung und Nachprüfung werden aber nur einzelne Spezialärzte angeregt, die bereits eine große technische Erfahrung hinter sich haben, während für die weniger Geübten die bloße Aufzählung einzelner Schlagworte eine Anregung nicht gewähren kann. Vor allem sind daher dem weniger Geübten Methoden zu übermitteln, auf deren Grundlage er weiter schaffen kann, ohne wie bei den schwierigen Methoden durch mangelnde Erfolge von vornherein enttäuscht zu werden.

Es soll nun in folgendem aus der Erfahrung heraus auf einzelne Methoden der Psychotherapie und ihre Verwendbarkeit für den Praktiker eingegangen werden. Es soll dabei auf neue Variationen, die sich für mich aus der Praxis ergaben und die auch für den allgemeinen Praktiker leicht zu erlernende Methoden darstellen, hingewiesen werden; auch einzelne Kunstgriffe, die die Handhabung der Methoden erleichtern und ihren Erfolg erhöhen, sollen zur Erwähnung kommen.

Die Erfahrungen entstammen meiner Tätigkeit an der Poliklinik des Kaiserin Auguste Victoria Hauses sowie meiner Privatpraxis. Zunächst ein paar Worte über die Beziehungen der Methoden zu den einzelnen Altersstufen des Kindes! Beim kleinsten Kinde kommt die Suggestion in einfachster Form zwecks Beruhigung zur Anwendung. 
C. Pototaky: Zur Methodik der Psychotherapie im Kindesalter etc. 105

Wir lauschen es der Mutter ab, wie sie mit einer gewissen Modulation in der Stimme und bestimmten, stets wiederkehrenden Lauten ihren Säugling beruhigt. Die alten Wiegenlieder weisen uns den Weg:

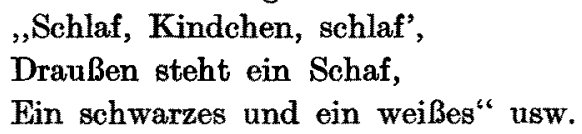

Wir sehen, wie hier die Zischlaute und die langen Vokale vorherrschen. Damit stimmt wohl auch der übliche Modus überein, ein Kind mit den gedehnten Lauten sch-sch-sch zu beruhigen. DaB em Kind auch in der entgegengesetzten Weise schon frühzeitig suggestibel ist, sehen wir sehr bald: es beteiligt sich rasch am Schreien, wenn es ándere Kinder schreien hört.

Kommt das Kind in das Spielalter, so kann man die eine oder andere psychotherapeutische Methode bereits vorsichtig zur Anwendung bringen. Daß die Autoren jedoch keineswegs hinsichtlich der Altersstufe, bei der man einzelne Methoden anzuwenden hat, einig sind, sieht man an den verschiedenen Beurteilungen, die die Hypnose nach dieser Richtung hin erfährt. Wenn z. B. Flatau an den Erfolg der Hypnose bereits bei 3 jährigen Kindern glaubt, sofern sie einigermaßen intelligent sind, so will F. Lust den frühesten Termin hierfür bei 5-6jährigen Kindern angesetzt wissen, während Kurt Singer sogar die Ansicht vertritt, daß die Hypnose wohl kaum vor dem 14. Lebensjahr anzuwenden sei. Auch ich selbst bin nach meinen Erfahrungen nicht dafür, zu kleine Kinder bereits einer hypnotischen Behandlung zu unterziehen. Als frühesten Termin für die Einleitung einer hypnotischen Behandlung möchte ich den Zeitpunkt nach den ersten Schuljahren bezeichnen, der also einem Alter von 8-10 Jahren entsprechen würde. Dieses Alter bildet meiner Erfahrung nach auch den Stützpunkt für die Anwendung der Mehrzahl der psychotherapeutischen Methoden.

Die Hypnose wende ich selbst in der letzten Zeit seltener an, einmal, da ich wie F. Ham burger der Ansicht bin, daß bei Kindern die Wachsuggestion einen größeren Einfluß ausübt wie die Schlafsuggestion, dann auch, weil mir ein unten zu beschreibendes, wachsuggestives Verfahren in einer großen Anzahl von Fällen, die ich früher mit Hypnose behandelt habe, gute Dienste leistet. Jedenfalls kann ein wachsuggestives Verfahren selbst bei etwa mangelhaften Erfolgen nicht schädigend wirken, wie ich es doch bei der hypnotischen Behandlung von Kindern gesehen habe. So denke ich an einen Fall von hartnäckiger Enuresis nocturna bei einem 10jährigen neuropathischen Knaben, der von den Eltern einem in der Hypnosenbehandlung sehr erfahrenen Kollegen 
zugeführt wurde. Die Enuresis verschlimmerte sich hier nach jeder Sitzung, vor allem geriet das Kind in derartige Erregungszustände, daß der Kollege nach wenigen Sitzungen den Abbruch der Hypnosenbehandlung anraten mußte. Die Verschlimmerung des Zustandes hielt jedoch nach der Beendigung der Behandlung noch lange Zeit hindurch an. Wenn natürlich derartige Fälle nicht zu verallgemeinern sind, so mahnen sie doch zur Vorsicht. Vor allem aber sollen wir uns nicht verleiten lassen, die Hypnose zu pädagogischen Zwecken anzuwenden oder gar ihre Anwendung Eltern und Lehrern, die an uns immer wieder mit diesbezüglichen Fragen herantreten, zu empfehlen. Hierin stehe ich auf dem gleichen Standpunkt wie L. Hirschlaff, der diese Frage einer ausführlichen interessanten Untersuchung unterworfen hat.

Wie gesagt, die Hypnose ist für mich bei der Behandlung von Kindern in der Mehrzahl der Fälle entbehrlich geworden, seitdem mir ein spezielles wachsuggestives Verfahren, das ich die „Milieusuggestions-Methode" nennen möchte, gute Dienste leistet. Diese Methode kann dem Praktiker sehr empfohlen werden, zumal da ihre Erlernung und Handhabung nach einiger Ubung keine Mühe macht: allerdings ist viel Liebe und sehr viel Zeit aufzubringen! Der die Methode beherrschende Arzt muß die Dialektik und Logik, wie sie zur Persuasion notwendig ist, mit der Konzentration verbinden, die für die Ausübung der Hypnose die unumgängliche Basis bildet. Natürlich läßt sich ein bestimmtes Sohema nicht aufstellen, da individuelle Gesichtspunkte, die sich aus dem Charakter, Bildungsgrad, Erziehung und Lebensverhältnissen ergeben, stets an die erste Stelle zu setzen sind. Daher hat man zunächst vorsichtig und unaufdringlich nach all diesen Punkten zu forschen, am besten in unauffälligem Gespräch mit dem Kinde und dessen Eltern. Hat man den Eindruck, als habe das Kind Vertrauen gefaßt, so werden die Eltern herausgeschickt. Das Kind bleibt mit dem Arzt allein, in bestimmten Fällen wird man eine „neutrale "Person der Sitzung beiwohnen lassen.

Ich lege nunmehr den kleinen Patienten auf ein Sopha, lasse ihn ruhig auf Kommando atmen und sage ihm, daß er jetzt geheilt werden wird. Er brauche nicht ängstlich zu sein, im Gegenteil werde er sich bald sehr beruhigt fühlen. Eventuell kann man, wenn das Kind lebhaft ist, womöglich - meist nur bei der ersten Sitzung - mit $Z$ wischenfragen kommt, einige der bei der Hypnose üblichen Sätze sprechen. „Du fühlst, wie Du ruhiger wirst", u. ä. Nach dieser Einleitung geht man zur eigentlichen Methode über, die ich an einem bestimmten Fall demonstrieren möchte: 
Knabe W. D., 10 Jahre alt, poliklinische Aufnahme 1953/18. Das $K$ nd ist das einzige Kind einer Lehrersfamilie, anscheinend verwöhnt, lelhaft. Vor 4 Monaten Grippe, seitdem auffallendes Nachschleppen des rèchten Beines. Dieses Hinken erscheint nach Angaben des Vaters unbeeinflußbar. Die Eltern sind daher besorgt und vermuten ein organisches Leiden.

Die Untersuchung des Kindes ergibt keinerlei Anzeichen einer Parese oder sonstigen organischen Nervenstörung. Die Patellarreflexe sind von normaler Stärke, beiderseits gleich. Babinski, Oppenheim, Mendel-Bechterew sind negativ, keine Spasmen. Aktive und passive Bewegungen im Bereich der unteren Extremitäten sind frei. In der Tat bemerkt man beim Gehen ein auffallendes Nachziehen des rechten Beines. Bei Ablenkung der Aufmerksamkeit oder bei Ermahnungen erfolgt hierin keine Änderung:

Es wird nun sofort die "Milieusuggestions-Methode“ eingeleitet:

Das Kind wird in der oben beschriebenen Weise hingelegt, ihm erklärt, daß es geheilt werden soll. Das Zimmer wird leicht verdunkelt, der Arzt setzt sich neben den Knaben ans Kopfende und suggeriert nunmehr mit einer gewissen Modulation in der Stimme, die für die Erhöhung der Suggestibilität unbedingt erforderlich ist. Die Stimme muß wohl monoton-beruhigend sein, muß aber doch bestimmte Stellen des Vortrages zu unterstreichen suchen, in einer leicht-gedehnten Betonung. Dabei darf natürlich die Stimme nicht so stark erhoben werden, da $B$ das Kind emporschrickt und aus seiner suggestibelen Vorstellung herausfällt.

Der Text der Suggestion lautete in vorliegendem Falle etwa folgendermaßen, nachdem einige einleitende Worte (wie oben beschrieben) vorausgegangen waren:

„Denke Dir, Du lägst auf einer hochgelegenen Bergwiese - - an einem schönen Sommernachmittag - - - die Sonne brennt warm _- _ behaglich dehnst und streckst Du Dich _ _ _ alles ist friedvoll, ruhig, so ruhig - - Du fühlst, wie die Sonnenstrahlen durch den Körper rinnen, ein angenehmes, köstliches Gefühl des Durchwärmtseins geht durch Deinen Körper. Von diesem lebenbringenden Wärmespender werden alle Teile des Körpers gleichmäßig getroffen. Und so merkst $\mathrm{Du}$, wie auch durch das schwache Bein ein neuer Quell der Kraft rieselt, Genesung wird Dir werden - - - Der Tag geht zur Rüste, heilige Stille herrscht oben auf der Höh'. - - - Noch klingen weither aus dem Tale ferne Laute - - Kleine Punkte bewegen sich, die Landleute, 
die die Ernte heimbringen wollen. - - - Leise zittern Abendlieder durch die Luft, dazu erklingen fast nur wie ein Hauch die Töne der Abendglocke - - Die Vögel verstummen, nur vereinzelt gaukeln noch ein paar Schmetterlinge vorbei — - - auch in Dir wird alles mahiger, nachgiebiger - - - die Muskeln an Deinem kranken Bein werden weicher - - Die Spannung läßt nach, mehr und mehr, alle Bewegungen stehen Dir wieder zu Gebote - - - Steh auf und freue Dich der wiedergewonnenen Gesundheit!"

In diesem Falle war das Hinken nach der Sitzung fast gänzlich geschwunden. Als sich der kleine Patient wenige Tage später wieder vorstellte, war das Gehen völlig normal. Spontan erklärte der Knabe, daß er sich vor allem freue, wieder "rennen" zu können. Das Stehen auf dem rechten - bisher ,kranken" - Bein gelang vollständig, auch die komplizierten Utbungen (z. B. Kniebeuge) wurden ohne Mühe ausgeführt, während vor der Sitzung die Kniebeuge ein sofortiges Zubodenfallen des Patienten hervorgerufen hatte. Das Kind wurde als geheilt entlassen. Natürlich wurden den Eltern entsprechende Aufklärungen gegeben, dem kleinen Patienten gegenüber dagegen läßt man sich möglichst wenig auf Exklärungen ein.

Von diesem Beispiele ausgehend soll noch auf einige Punkte näher eingegangen werden. So muß auch bei dieser psychotherapeutischen Methode der Nachdruck auf ein sicheres und zielbewußtes Auftreten des Arztes gélegt werden. Es ist für den Ungeübten nicht ganz leicht, selbst dem Kinde gegenüber „Herr der Situation“ zu bleiben. Das Kind durchschaut sofort jede Unsicherheit, und der Kontakt ist gelöst. Man lasse sich nicht durch das verlegene oder erregte Lachen stören, das besonders während der ersten Sitzung beim Kinde hervortritt. Zwischenrufe und -fragen weise man ruhig mit der Bemerkung zurïck, das Kind dürfe jetzt nicht sprechen, sondern müsse erst ruhig zuhören. Das Kind muß eben zunächst in eine gewisse Suggestibilität versetzt werden; es muß Vertrauen zu der Persönlichkeit des Arztes gewinnen.

Vor allem ist. die Wirkung jedes einzelnen Wortes auf das Kind scharf zu kontrollieren. Stets ist darauf zu achten, daß dabei die Phantasie nicht übermäßig angeregt wird: es darf eben keinesfalls statt der Beruhigung und einer gewissen Erschlaffung ein aufgeregtes „Aufhorchen" erzielt werden. Man erkennt es an plötzlichem Heben der Augenlider und einem sich straffenden Muskelspiel, wenn gefühlsbetonte Komplexe getroffen werdeu. Dann muß man rasch dem Thema eine andere Wendung geben und in besonders ausgeprägt-monotoner Sprechweise dem Inhalt den erregenden Reiz zu nehmen suchen. Gerade bei älteren Kindern 
darf man das ,gefühlsneutrale" Gebiet nach Möglichkeit nicht verlassen; bei den dem Pubertätsalter sich nähernden Altersstufen hat man besonders scharf aufzupassen.

Was die Landschaftsbilder betrifft, in die man das Kind versetzt, so hat man darauf zu achten, daß man dem Kinde möglichst bekannte Bilder vorführt, etwa Motive, die an den letzten Ferienaufenthalt anklingen. Nur ist, wie gesagt, das Anschlagen stark gefühlsbetonter Komplexe nach Möglichkeit zu vermeiden. Aus ähnlichen Gründen tut man gut, einen krassen szenischen Wechsel in ein- und derselben Sitzung zu vermeiden. Denn analog scheint auch bei den gewöhnliohen Märchenerzählungen gerade der szenische Wechsel die. Phantasie des Kindes besonders anzuregen, wie wir dies aus den Versuchen, die Char lotte Bühler bei Märchenerzählungen angestellt hat, ersehen.

Hinsichtlich des frühesten Alterstermins, bei dem die Anwendung der "Milieusuggestions-Methode“ zu empfehlen wäre, ist zu bemerken, daß man bei einem verständigen 5 jährigen Kinde wohl bereits den Versuch machen kann, also bereits zu einem früheren Zeitpunkte, als ich ihn oben bezüglich der Einleitung einer hypnotischen Behandlung bezeichnet hatte.

Die Frage, ob man das gleiche Bild bei den späteren ,,milieusuggestiven" Sitzungen zu verwerten habe, wie man es für die erste Sitzung benutzt hatte, läßt sich nicht einheitlich beantworten. In der Regel empfiehlt es sich, bei jüngeren Kindern dasselbe Bild zu wiederholen, bei älteren Kindern neue Bilder oder wenigstens neue Mosaikstücke im alten Rahmen einzuschieben. Denn wir sehen bei Märchenerzählungen in täglicher Erfahrung, daß das kleine Kind immer und immer wieder dasselbe Märchen gern hört und es sich stets wiederholen läßt, ohne nach neuen Märchen zu verlangen, während das ältere Kind bei Wiederholung des gleichen Stoffes nicht mehr aufmerkt, sondern seine Gedanken schweifen läßt.

Weiterhin ist namentlich bei älteren Kindern darauf zu achten, daß sie nicht ihre Gedanken gerade durch die sie interessierende Behandlung auf ihre Krankheit zu stark konzentrieren. Bemerkt man dies, so hat man durch eine Änderung der Art und der Zahl der Sitzungen dieser Gefahr zu begegnen. Jedenfalls ist die Gefahr der zu intensiven Hinlenkung auf das eigene Ich bei dieser Methode viel geringer als bei den meisten anderen. Diese Gefahr hält $u$, a. Allers bei der Psychoanalyse im Kindesalter für besonders groß.

Noch einige Worte über einen kleinen Kunstgriff, den ich bei dieser Suggestionsmethode zur Erhöhung der Suggestibilität mit Erfọlg bei 
Kindern anwende und der auch für die Suggestionstherapie bei Erwachsenen und bei der Hypnose zu empfehlen ist: Ich lasse während der Sitzung die Kinder mit einem Kittel, etwa in Form eines weißen Schwesternmantels, bekleiden und habe dabei beobachtet, daß die Kinder bereits durch das ihnen ungewohnte Kleidungsstück in eine fast feierliche, jedenfalls für neue Eindrücke zugänglichere Stimmung geraten. Diese Erfahrung ist ähnlich der in Kriegslazaretten gemachten und, wie ich glaube, $u$. a. von Nonne angegebenen Beobachtung, nach der die Soldaten der Beeinflussung bei der Hypnose in entkleidetem Zustande zugänglicher wurden. Wenn ich nun scheinbar das umgekehrte Verfahren anwende, so erziele ich doch die gleiche Wirkung, da das Alltagsgewand in beiden Fällen eine Änderung erfährt und dadurch der Träger desselben in einen von vornherein unselbständigen Zustand versetzt wird.

Die "Milieusuggestions-Methode" ist nach dem vorher Gesagten ein wachsuggestives Verfahren, das auf folgenden Gesichtspunkten beruht: Das Kind soll möglichst ohne Hypnose in einen suggestiblen Zustand versetzt werden. Um dies zu erreichen, wird eine Milieuänderung dem Kinde in der Suggestion gleichsam als Bild vorgeführt; auf diesem Boden, der gewissermaßen dem Alltäglichen entrückt ist, wird nun das Kind für die suggestiven Ziele, die der Arzt für wünschenswert hält, empfänglicher gemacht, und zwar habe ich den bestimmten Eindruck, daß gerade diese ,Milieuänderung in der Suggestion" den günstigen Boden abgibt, da ich mit Suggestion in anderer Form, z. B. beruhigenden, lehrhaften Inhalts, nicht die gleichen Erfolge erziele. Die Bewertung der Milieuänderung an sich ist bekanntlich umstritten. Czerny hat - ich stimme darin völlig mit ihm überein in dankenswerter Weise energisch gegen die ,Milieuänderung um jeden Preis" bei neuropathischen, schwer erziehbaren Kindern Front gemacht und der Behandlung und Erziehung zu Haus das Wort geredet. In der Tat überwiegen oft die Nachteile der Milieuänderung deren Vorteile. Andererseits sind die günstigen Erfolge der Milieuänderung in gewissen Fällen nicht zu leugnen - Kinder sind eben „Milieumenschen“, wie H. Vogt bemerkt - und so habe ich die Vorteile der Milieuänderung in der Milieusuggestions-Methode nutzbar gemacht, ohne die Kinder dem Elternhause entziehen zu brauchen. Denn schon die „Milieuänderung in der Suggestion" bietet eine mächtige Ablenkung! Das Kind, das sich auf die weite stille Heide, an den Meeresstrand mit dem Ausblick auf die leise plätschernden Wogen, aus dem Häusermeer der Großstadt hinwegträumt - ich will hier zunächst nur den örtlich-landschaftlichen Charakter des Milieus ins Auge fassen - wird dadurch aus dem Alltags- 
einerlei herausgerissen und von seinen Vorstellungen abgelenkt. Damit ist die Basis für die aktive Beeinflussung seitens des Arztes gegeben!

Aber auch wichtige Aufschlüsse vermag uns die ,Milieusuggestions-Methode“ zu vermitteln! Sie gibt eine Vorprüfung ab, wie ein tatsächlicher Aufenthaltswechsel, der für schwierige Fälle als letztes Hilfsmittel doch noch in Frage kommen wird, auf den kleinen Patienten wirken würde! Bei einiger thbung gelingt es leicht, in der Sitzung aus dem Gesichtsausdruck des Kindes sowie aus den Reflexbewegungen herauszulesen, in welcher Stärke der Milieuwechsel zu wirken vermag und wie ein tatsächlicher Wechsel demnach wirken würde, ferner welche Reizworte einen Gefühlskomplex treffen, und so kann man auch für die Wahl des Aufenthaltsortes wichtige Weisungen erhalten. Jedenfalls kann die Gefahr einer unrichtigen Milieuwahl, die doch für das Kind nicht gleichgültig ist, erheblich herabgemindert werden.

Noch einige Worte über die Indikationen, wie sie für die Anwendung der "Milieusuggestions-Methode" aufzustellen sind. Zur Behandlung geeignet für diese Methode sind die Äußerungen des neuropsychopathischen Kreises. Im Vordergrunde steht die Behandlung der Angstzustände; hier scheinen die Versuche besonders günstige Resultate zu ergeben. Dabei kann ich nicht F. Hamburger beipflichten, der die Erfolge der Wachsuggestion bei den bei Tage sich zeigenden psychogenen Erscheinungen für ungünstiger hält als jene bei abnormen Schlafgewohnheiten. Denn ich kann nach meinen Erfahrungen, wenigstens hinsichtlich der „Milieusuggestions-Methode“, keinen Unterschied in der Wirkung auf Tages- und Nachtstörungen feststellen, eher noch einen solchen zugunsten einer leichteren Beeinflussung der Tageserscheinungen.

Außer bei den eigentlichen Angstzuständen ist die Methode bei psychogenen Lähmungen und Angewohnheiten, bei Schlafstörungen, Enuresis und ähnlichen Zuständen heranzuziehen. Ebenso bringe ich die psychische Behandlung, wie sie Czerny und Keller bei den vasomotorisch-blassen, unterermährten Kindern empfiehlt, in Form der „Milieusuggestion" zur Anwendung.

So viel über die "Milieusuggestions-Methode". Sie bietet eine gute Grundlage für die psychische Behandlung im Kindesalter, ist leicht zu erlernen und individuellen Modifikationen zugänglich.

Den Kindern und Eltern gegenüber hat die Methode den Vorzug, daß sie des mystischen Charakters entbehrt, ein Vorzug, der der Hypnose, der Psychoanalyse und anderen Methoden namentlich seitens der Eltern nicht ohne weiteres zugebilligt wird.

Natürlich darf diese Methode niemals als die Methode $K \alpha \boldsymbol{x}^{\prime} \boldsymbol{\varepsilon} \xi o \chi \eta^{\prime} \boldsymbol{v}$ 
angesehen werden - ebensowenig wie irgend eine andere psychothera peutische Methode! Leider wird unter den Psychotherapeuten gegen diesen Grundsatz oft vergangen; jeder ist nur auf "seine" Methode eingestellt und unterwirft ihr sämtliche seiner Patienten. Wenn ich auch der Ansicht bin, daß nicht nur der Psychotherapeut die Methode sucht, sondern vielmehr die Methode den Psychotherapeuten, und daß hierdurch eine gegenseitige intensive Verankerung stattfindet; so muß doch diese „Einstellung" überwunden werden und in psychologischem Erfassen diejenige Methode stets von neuem gesucht werden, die für den einzelnen Fall geeignet ist. Es ist dies keine bequeme Aufgabe, aber die Mühe wird durch die Erfolge belohnt werden.

\section{Iiteraturverzeichnis.}

1. Allers, Diskussionsbemerkungen. Münch. G̣esellsch. f. Kinderheilk, Sitzg. vom 27. März 1914.

2. Bühler, Charlotte, Das Märchen und die Phantasie des Kindes. Verlag von Joh. Ambros. Barth, Leipzig, 1918. (Beihefte z. Zeitschr. f. angew. Psychologie.)

3. Czerny, Adolf, Das schwer erziehbare Kind. Jahrbuch f. Kinderheilk. 85, Heft 4. 1917.

4. Czerny, Ad., und A. Keller, Des Kindes Ernährung, Ernährungsstörungen und Ernährungstherapie. Ein Handbuch für Ärzte. 8. Mitteilung. Leipzig u. Wien 1913, Franz Deuticke.

5. Flatau, Georg, Kursus der Psychotherapie und des Hypnotismus. Berlin 1918 , S. Karger.

6. Goett, Psychotherapie in der Pädiatrie. Münch. med. Wochenschr. 1914, Nr. 25.

7. Hamburger, Franz, Schlafstörungen im Kindesalter. Monatsschr. f. Kinderheilk. 13, 1916.

8. - Utber Psychotherapie im Kindesalter. Wiener med, Wochenschr. Jahrg. 64. Nr. 24. 1914.

9. Hirschlaff, L., Suggestion und Erziehung. Berlin 1914, J. Springer.

10. Löwenfeld, L., Zum gegẹnwärtigen Stande der Psychotherapie. Münch. med. Wochenschr. 57, Nr. 3/4. 1910.

11. Lust, F., Diagnostik und Therapie der Kinderkrankheiten. Berlin 1918 , Urban \& Schwarzenberg.

12. Oppenheim, H., Lehrbuch der Nervenkrankheiten. Berlin, S. Karger.

13. Schultz (Jena), Wege und Ziele der Psychotherapie. Therap. Monatshefte. Jahrg. 29. 1915.

14. Singer, Kurt, Die Schreckneurosen des Kindesalters. Jena 1918, Gustav Fischer.

15. Vogt, H., Psychotherapie. Therap. Monatshefte. 27, 1913.

16. - Handbuch der Therapie der Nervenkrankheiten. 1. Jena 1916, Gustav Fischer. 